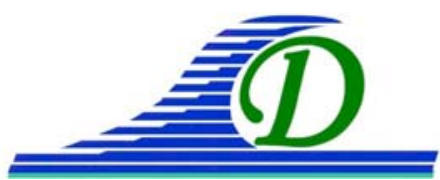

XIII ${ }^{\text {ìmes }}$ Journées Nationales Génie Côtier - Génie Civil

Dunkerque, 2-4 juillet 2014

DOI:10.5150/jngcgc.2014.007 C Editions Paralia CFL

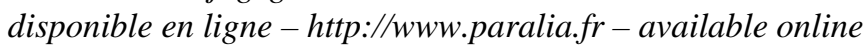

\title{
Base de données courantométriques au point fixe du SHOM
}

\author{
Stéphanie DESMARE ${ }^{1}$, Pascal LE DU ${ }^{1}$
}

1. Service hydrographique et océanographique de la marine,

Département hydrodynamique côtière (HDC) - Courants, SHOM/DOPS/MIP/HDC, 13, rue du Chatellier, CS92803, 29228 Brest Cedex 2, France.

stephanie.desmare@shom.fr ; pascal.ledu@shom.fr

\section{Résumé :}

Le SHOM détient une grande partie des observations des courants marins au point fixe réalisées aux abords des côtes françaises et d'outre-mer. Elles sont en général effectuées pour les besoins de l'hydrographie, dans les sites intéressants pour la navigation maritime ou les besoins scientifiques. Les séries temporelles de courantométrie archivées au SHOM ont été collectées soit par ses propres groupes hydrographiques soit par des organismes extérieurs. Elles constituent la source essentielle des informations relatives aux courants de marée figurant sur les cartes marines et dans les ouvrages nautiques (instructions nautiques, etc.). Ces données sont également utilisées pour la validation des modèles numériques de courant (utilisés pour les atlas de courants de marée) et la réalisation des prestations spécifiques (expertise, soutien aux forces, soutien aux politiques et acteurs de la mer et du littoral).

Le besoin de rénovation de la gestion des mesures de courants marins au SHOM a été défini dans le cadre d'un projet thématique INFRAGEOS-H qui a pour but de constituer une infrastructure géospatiale c'est-à-dire un système d'enregistrement et d'accès numérique aux informations géospatiales hydrographiques du SHOM.

La refonte de la base de données in situ de courants au point fixe est en cours de finalisation. Le nouveau système qui est présenté dans l'article, intègre ainsi l'ensemble des données de courants marins disponibles sous forme numérique (séries brutes à cadence d'acquisition, données historiques numérisées et séries validées) et données auxiliaires (pression atmosphérique, températures, vent,...). La nouvelle base Oracle contient dorénavant les métadonnées, décrivant notamment les campagnes d'observation et instruments, et la documentation associée sous forme de dossiers de courant.

Mots-clés : Génie côtier, Hydrodynamique côtière, Marée, Courant, Instrumentation, Base de données.

\section{Introduction}

L'observation et l'acquisition de la connaissance des courants au point fixe présentent plusieurs intérêts et sont justifiées par de nombreux besoins comme : les besoins de l'océanographie nautique (sécurité de la navigation, homogénéiser la répartition spatiale 


\section{Thème 1 - Hydrodynamique côtière}

des observations pour la modélisation), les besoins en océanographie militaire (au profit des opérations amphibies ou de guerre des mines), la modélisation hydrodynamique, les besoins pour le soutien des politiques publiques (besoin de connaissance plus générale le long et au voisinage des côtes, études environnementales, développement des énergies marines renouvelables, aménagement et dimensionnement des ouvrages).

La refonte du système d'archivage et de gestion des mesures de courants au SHOM a été nécessaire et intégrée à un projet plus vaste de réalisation d une infrastructure de données géospatiales baptisé projet INFRAGEOS-H.

Ce projet INFRAGEOS-H, débuté en 2007, a permis la rénovation de la base de données bathymétriques, de la base de données générales (balisage, amers, épaves, obstructions, zones réglementées...) et enfin de la base des données marégraphiques du SHOM.

Concernant la gestion de ces différentes bases de données, le SHOM utilise le module Source Editor de la suite logicielle Hydrographic Production Database (CARIS HPD). Ce système permet l'affichage cartographique et la visualisation des couches géographiques nécessaires pour parcourir les données (trait de côte, zones de marée, cartes marines, etc).

Pour les données de courants et les outils qui lui sont actuellement associés, il a fallu trouver un système d'interfaçage de la base avec l'infrastructure géospatiale du SHOM. Un développement spécifique était donc nécessaire pour la création du module de stockage, de gestion, de visualisation et de traitement des mesures courantométriques. Le développement de ce module a été confié à la société anglaise Chersoft Ltd et baptisé TideCurrentDataBase (TCDB).

\section{Existant}

\subsection{Instruments}

Les séries temporelles de vitesses et directions des courants sont issues de tous types de courantomètres immergés au point fixe (courantomètres mécaniques, courantomètres électromagnétiques, courantomètre acoustiques). Les courants sont soit mesurés à l'immersion du courantomètre (ex : courantomètre à rotor, ou courantomètre ponctuel Doppler) soit dans des couches d'épaisseur prédéfinies sur toute la colonne d'eau (profileur ADCP).

\subsection{Traitement et archivage}

Les données de courant sont traitées et validées par des logiciels généralement spécifiques (constructeur ou développés en internes) :

pour le traitement des mesures issues des courantomètres ponctuels (SUBER SLS21, MORS MC360, SONTEK ARGONAUT, InterOcean S4, FSI 3DACM, NORTEK AQUADOPP), 


\section{XIII ${ }^{\text {èmes }}$ Journées Nationales Génie Côtier - Génie Civil \\ Dunkerque, 2-4 juillet 2014}

- pour le traitement des profileurs de courants à effet Doppler des constructeurs Nortek (modèle Continental, Aquapro, NDPO, ADP et AWAC) et Teledyne-RDI (modèle ADCP Workhorse et compatible), immergés au point fixe et orientés vers le bas ou vers le haut.

Toutes les mesures issues de mouillages sont disponibles sous forme de fichiers numériques bruts archivés dans des répertoires dédiés. Elles constituent la source essentielle des informations relatives aux courants de marée figurant sur les cartes marines et les ouvrages nautiques (atlas de courant de marée, instructions nautiques, ...). Les données sont échantillonnées à pas de temps constant. Pour la plupart, leur traitement et validation au SHOM a donné lieu à la constitution d'un archivage dans un fichier numérique unique contenant, lorsque le courant de marée était prédominant, les résultats de calcul de courants en vive-eau moyenne et en morte-eau moyenne par rapport aux heures de marée, ainsi que des tableaux de constantes harmoniques de courants et courants moyen.
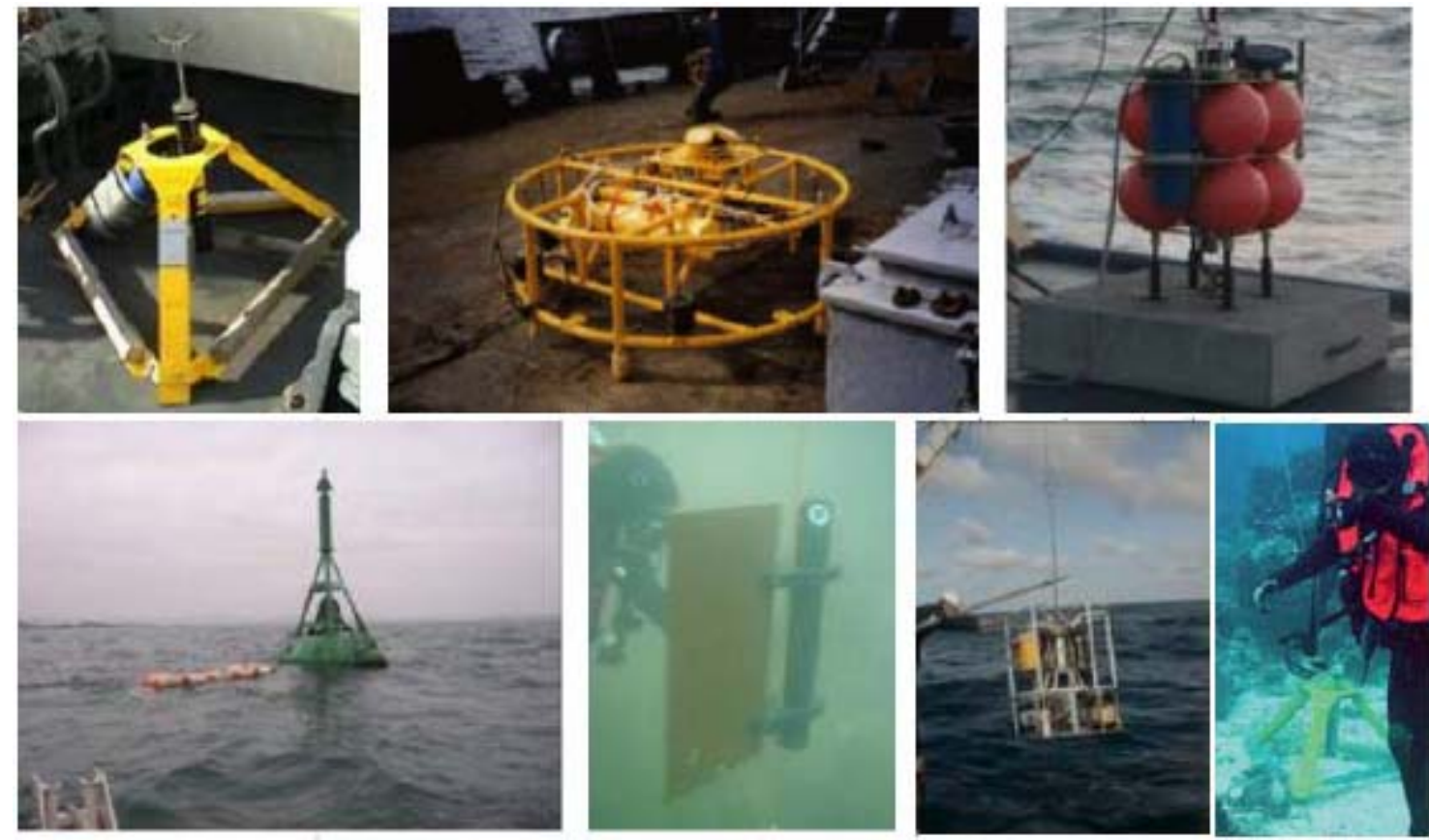

Figure 1. Les appareils et les différents modes d'utilisation.

\subsection{Informations disponibles}

La base TCDB est dédiée aux données de courants au point fixe (données issues des courantomètres au point fixe, courantomètres Doppler ponctuels et profileurs).

Les données issues de calcul sur modèle numérique (en 2D et 3D) ne sont pas contenues dans la base compte tenu de l'abondance que représentent potentiellement ces données. Il a toutefois été rendu possible d'exploiter ces résultats en les chargeant via les outils de traitements spécifiques qui ont été développés dans l’interface d’accès à la base. 
Du fait de leur volumétrie et de leur gestion dans d'autres bases de données, les mesures de courantométrie issues des bouées dérivantes, les mesures acquises en route ou par radar HF ne sont pas prises en charge dans cette base.
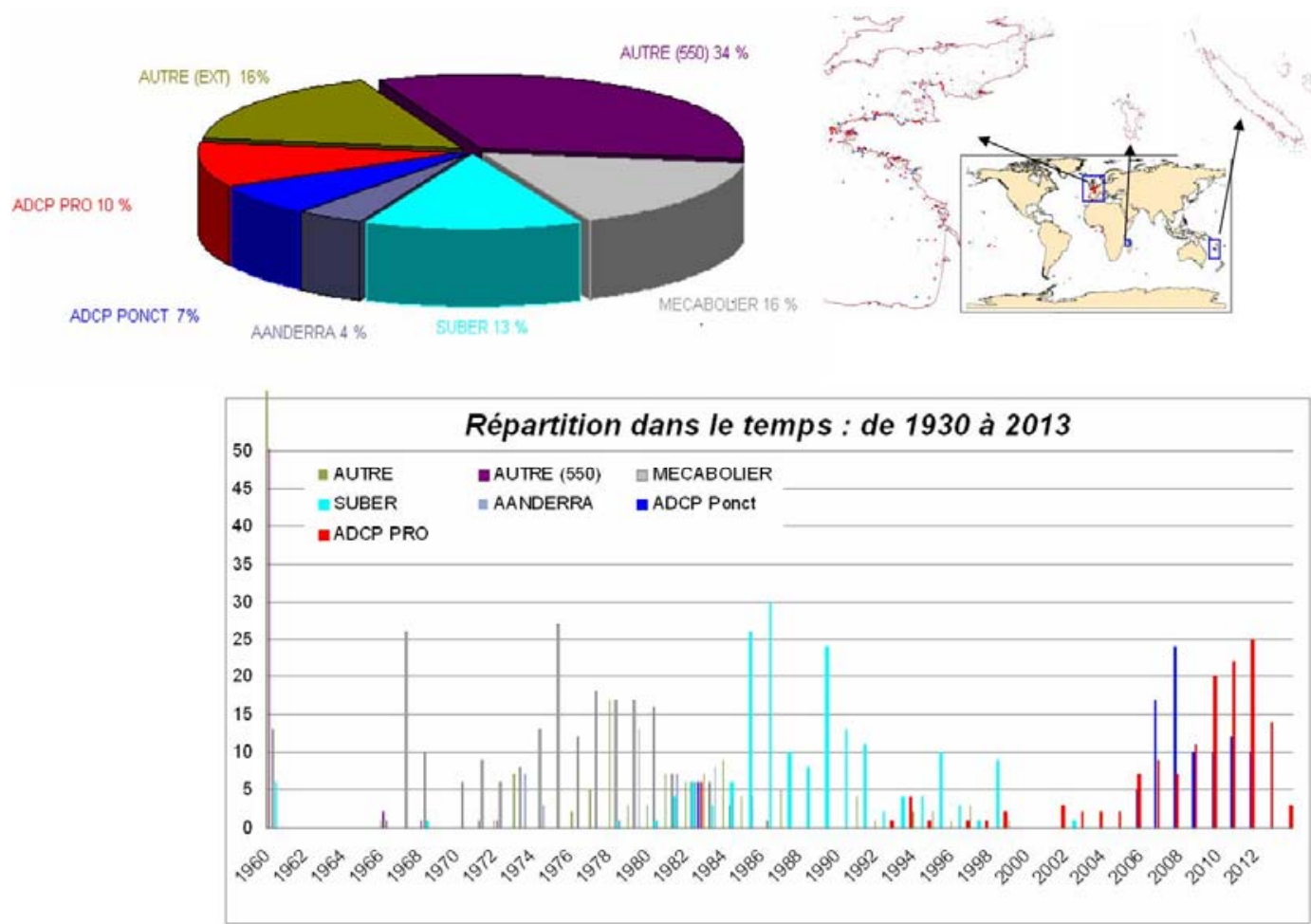

Figure 2. Une vue globale des informations disponibles.

\section{Refonte de la base de données de courants au point fixe}

Le besoin a été fortement identifié de disposer d'un ensemble d'outils qui permette d'archiver, de visualiser, de qualifier et d'exploiter les données de courants au point fixe, d'avoir une meilleure traçabilité et de rendre la consultation de données disponibles et leur mise à disposition aisées pour les utilisateurs de toute nature au SHOM.

Les développements et spécifications de la nouvelle base ont démarré en 2012 et seront finalisés courant 2014 par l'admission du système suite à la migration de l'existant et mise en production du système.

\subsection{Architecture du système}

Le logiciel Henry (développé par la société Chersoft) assure une interface avec TideCurrentDataBase et propose une couche comprenant les stations de courants et leurs métadonnées. TideCurrentDataBase archive toutes les informations et données relatives aux stations de courants dans une base de données Oracle. 


\section{XIII ${ }^{\text {èmes }}$ Journées Nationales Génie Côtier - Génie Civil \\ Dunkerque, 2-4 juillet 2014}

Les modules d'administration (TDB Business Administration Utility) et d'export des données (TDB Export Wizard) sont deux applicatifs indépendants du logiciel de SIG (voir figure 3).

Les principales fonctions d'édition et de visualisation de TCDB peuvent alors être appelées depuis ce TdBEditor (voir figure 4).

Tableau 1. Etapes de réalisation du système.

\begin{tabular}{ll}
\hline & Objectif \\
\hline Phase de conception & Création d'une base qui regroupe toutes les données et \\
& métadonnées courantométriques au point fixe existantes au \\
& SHOM interfaçage de la base avec l'infrastructure géospatiale \\
& du SHOM (le système d'information géographique utilisé pour \\
& les autres thèmes de l'infrastructure est CARIS HPD Source \\
& Editor) \\
Développement des fonctionnalités & Outils graphiques de visualisation et manipulation, accès pour \\
indispensables à la gestion et & enregistrement, catalogage, recherche, export \\
l'exploitation de la base & \\
Développement de fonctionnalités & Visualisation graphique et enregistrement de roses de courant, \\
liées à des outils de traitement plus & réalisation et archivage des tableaux de courants de marée, \\
spécifiques aux besoins de & analyses statistiques des mesures, utilisation des données pour \\
production du SHOM & la validation de modèles, exports en différents formats, \\
& recherches thématique \\
Développement des outils de & Migration des données de courant anciennement archivées en \\
migration & assurant la compatibilité avec les applications SHOM \\
& utilisatrices de ces données
\end{tabular}

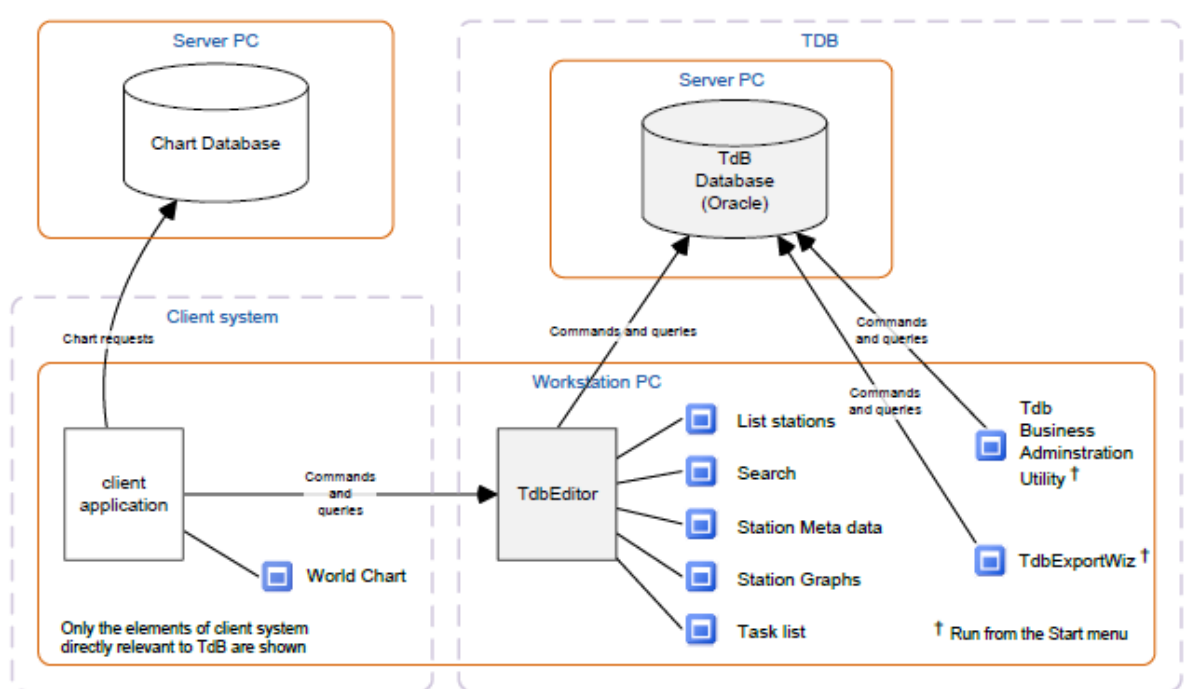

Figure 3. Architecture du système (schéma Chersoft). 


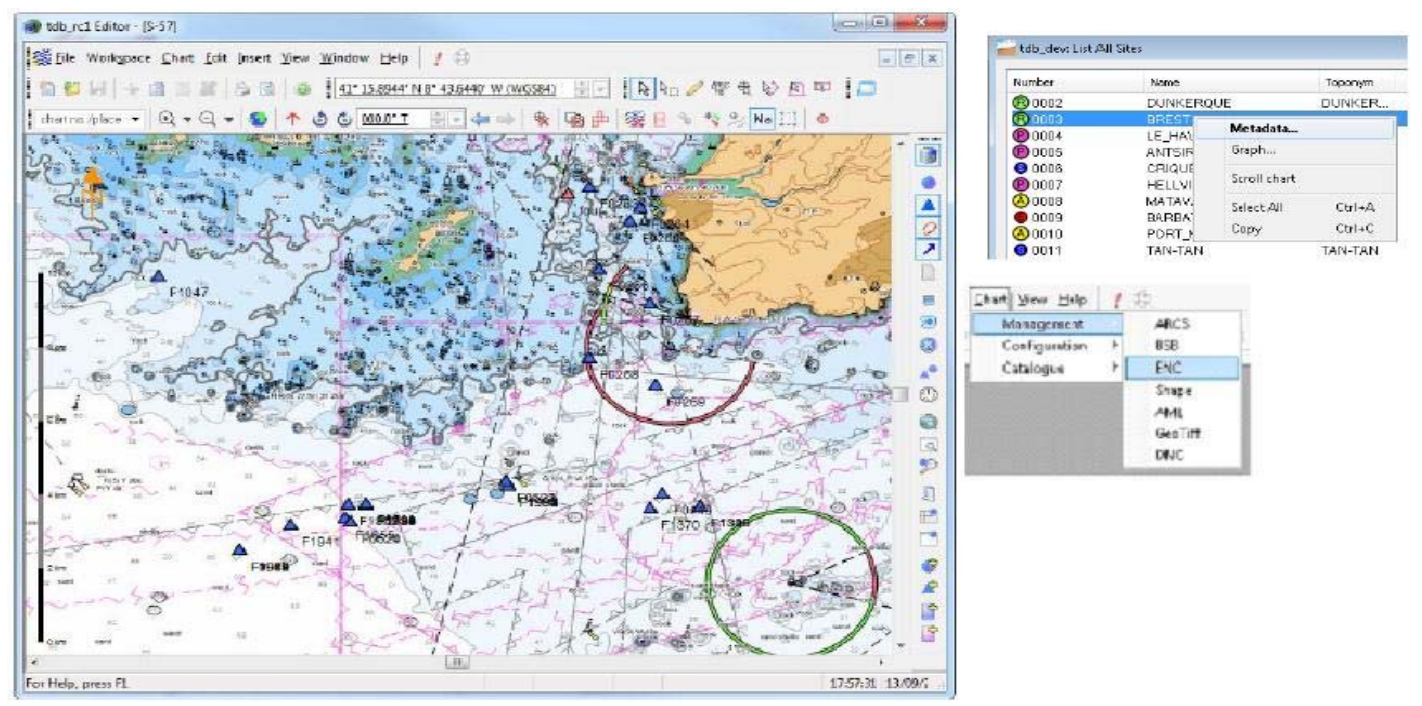

Figure 4. Elements de cartographie et de visualisation via l'interface Henry pour accéder à la base de données courant.

\subsection{Workflow}

Les tâches réalisées par les différents intervenants dans la base de données comprennent l'archivage et la saisie des métadonnées, le traitement et la validation, puis le contrôle et l'approbation. Ces travaux sont réalisés par les entités du SHOM impliquées au travers d'un processus métier (workflow), qui assure que seule la donnée validée et approuvée est disponible à l'ensemble des utilisateurs.

\subsection{Attribution des rôles et des droits}

Les droits sont administrés dans une interface spécifique et configurés pour chaque utilisateur. Les droits disponibles à l'attribution sont : la consultation, l'édition, la finalisation d'une campagne de mesure, l'approbation d'une station de courant, l'export des données de la base ou encore l'administration du système.

\section{Résultats}

Le nouveau système remplace donc les anciens outils de gestion et d'archivage et contient toutes les informations, notamment l'ensemble des données de courants marins disponibles sous forme numérique (séries brutes à cadence d'acquisition, données historiques numérisées et séries validées) et données auxiliaires (pression atmosphérique, températures, vent, ...). La nouvelle base Oracle contient les métadonnées, décrivant notamment les campagnes d’observation et instruments, et la documentation associée sous forme de dossiers de courant sous format numérique.

Les principales fonctionnalités de TideCurrent DataBase sont : 


\section{XIII ${ }^{\text {èmes }}$ Journées Nationales Génie Côtier - Génie Civil \\ Dunkerque, 2-4 juillet 2014}

- l'archivage et la validation des résultats de campagnes de mesures courantométriques incluant les données instrumentales et les informations géodésiques associées,

- la validation des données de courant en relation avec les données annexes, les données antérieures et les données acquises simultanément à proximité,

- la consultation et la mise à disposition de ces informations.

- les outils de traitement des données pour le calcul des tableaux de courant de marée reportés sur la plupart des cartes marines,

- l'édition du dossier de courant et mise en forme des métadonnées,

- la visualisation des métadonnées,

- la visualisation des données avec différents modes de représentation (tracés graphiques et de tableaux). Les fichiers bruts tels qu'ils ont été importés dans la base sont aussi visualisables,

- la recherche multicritère en particulier pour la recherche de station de courant, d'une campagne de mesure, d'instruments, de publication, etc.,

- l'export des données avec une interface indépendante (TDB Export Wizard) qui permet l'export sous forme de fichiers aux formats génériques mais aussi spécifiques de tous les types de données disponibles dans la base (seulement pour la dernière version approuvée de la ou des stations de courant sélectionné(es)).

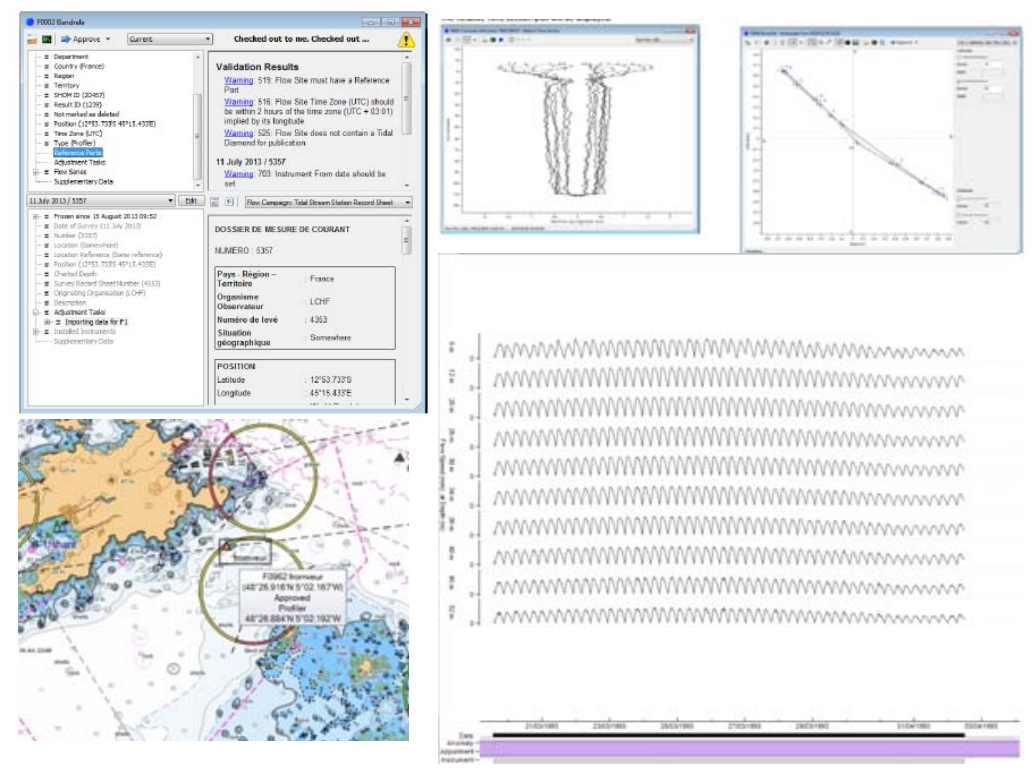

Figure 5. Exemple de fonctionnalités offertes par l'éditeur Henry et interface d'accès à la base.

\section{Perspectives}

Une étape importante du projet sera la fiabilisation de la base par la vérification de la complétude des données migrées et de leurs métadonnées. 
Une autre étape du projet consistera à la publication des métadonnées de cette base sur Internet pour les zones sous juridiction nationale, dans le cadre particulier de la directive INSPIRE (CE, 2007), le portail data.shom.fr assurant, de fait, la diffusion des données géographiques maritime et littorale.

\section{Conclusion}

L'outil TideCurrentDataBase permettra dès sa mise en service une gestion intégrée et efficiente du patrimoine. La base accueille alors toutes les données issues des campagnes des groupes hydrographiques du SHOM. La base pourra aussi intégrer les données recueillies dans le cadre de convention avec des organismes extérieurs.

Le déploiement de l'outil s'achèvera en 2014 avec une mise en oeuvre dans les groupes hydrographiques, qui permettra le déroulement intégré du processus métier complet, allant de la rédaction à la validation des travaux courantométriques et leur diffusion. TideCurrentDataBase permet à un utilisateur d'accéder à la dernière version validée d'une station de mesure et de l'exporter, indépendamment des modifications en cours.

\section{Références bibliographiques}

CE, Directive 2007/2/CE du 14 mars 2007 établissant une infrastructure d'information géographique dans la Communauté européenne (INSPIRE). 